\title{
Model of poleward magnetic field streams from sunspot butterflies
}

\author{
Nadezhda V. Zolotova and Dmitri I. Ponyavin \\ Institute of Physics, St. Petersburg State University, \\ Ulyanovskaya ul.1, Petrodvorets, St. Petersburg, Russia, 198504 \\ email: ned@geo.phys.spbu.ru
}

\begin{abstract}
The poleward magnetic field streams on time-latitude diagram of the photospheric magnetic field of the Sun during 1975-2011 (Kitt Peak NSO, SOLIS NSO, SOHO MDI data) are modeled. We performed simulations in terms of probability density function and bipole orientation according to Joy's law and Hale's cycle. The difference between distributions of leading and trailing fluxes of bipolar sunspots defines the so-called surplus. Finally, magnetic field streams and polar field reversals are a result of meridional drift of a surplus to the poles.
\end{abstract}

Keywords. Sun: activity, Sun: magnetic fields, (Sun:) sunspots.

\section{Introduction}

The time-latitude diagram of the axisymmetric photospheric magnetic field of the Sun during 1975-2011 (based on Kitt Peak NSO, SOLIS NSO, SOHO MDI data) shows magnetic field streams (or surges — Wang, Nash \& Sheeley 1989) toward the poles (Hathaway 2010). It is believed that the intermittent structure of photospheric magnetic field is result of faster meridional flow which produces less unbalanced trailing-polarity flux, generates surges of leading polarity, and decreases the polar and heliospheric fields (Schrijver \& Liu 2008; Wang, Robbrecht, \& Sheeley 2009; Dikpati, Gilman, \& Ulrich 2010; Hathaway \& Rightmire 2011; Nandy, Muñoz-Jaramillo, \& Martens 2011). Thus the role of meridional flow in evolution of the Sun's large-scale field is emphasized. The role of diffusion is in annihilating leading flux at low latitudes (Wang, Nash \& Sheeley 1989).

Using the hypothesis for spatio-temporal organization of sunspot activity over the solar surface as impulses (Gnevyshev 1938; Antalová \& Gnevyshev 1983) we found a link between impulses and poleward magnetic streams (Zolotova \& Ponyavin 2012a). Each sunspot impulse causes the latitudinal drift of unbalanced flux of new polarity in each hemisphere, whereas the poleward streams of old polarity occur in gaps between impulses (Zolotova \& Ponyavin 2012b).

In this paper, we present simple model for magnetic surge reconstruction from impulses, analyze dependence of polar field on meridional flow profile and compare simulated poleward magnetic surges with the real observations.

\section{Model}

The bipolar magnetic regions (BMRs) tend to be "tilted" with the leading spot closer to the equator than the following spots (Joy's law). Hale et al. (1919) noted the increase of the average axial tilts of sunspot groups with latitude. This law is suggested as a result of the action of Coriolis forces on the rising magnetic flux tubes (Cowling 1965; Stenflo 
\& Kosovichev 2012). Mackay, Priest, \& Lockwood (2002) claimed that the total surface flux, total open flux, the total dipole and the nonaxisymmetric dipole depends crucially on the tilt of the BMR axis.

In axisymmetric case we processed matrixes with step in latitude $-1^{\circ}$, time step one Carrington rotation. Because of averaging over longitude, a separation of leading and trailing spots in latitude is defined not by means of tilt angle $\alpha$, but just by latitudinal segregation $\Delta l$ (in degrees of latitude):

$$
\Delta l=10 \tan (\alpha)=10 \tan (f \cdot l),
$$

where $l$ is latitude, $f=0.5$, according to Wang \& Sheeley (1989), Wang \& Sheeley (1991). Obviously $\Delta l$ as well as $\alpha$ increases toward high latitudes and vanishes equatorward.

Figure 1 schematically shows the modeling process performed in terms of probability density function (PDF). For simplicity we consider solar cycle as bivariate Gaussian in time-latitude plane. At first we generate leading spot PDFs in hemispheres (Fig. 1a). Yellow and blue define polarities. Major axes of PDFs are denoted by red. Trailing spot PDFs are generated by transformation of leading spot PDFs in view of Hale's and Jow's laws. We change polarity sign of PDF (multiplication by minus one) and add latitudinal segregation $\Delta l$ to latitudinal coordinate: $l_{\text {trail }}=l_{\text {lead }}+\Delta l(l)$. Major axes for trailing PDFs are denoted by black. Hence, density distributions of leading and trailing spots have slightly different coordinates in time-latitude plane. This difference vanishes equatorward, as it have to be according Eq. 2.1.
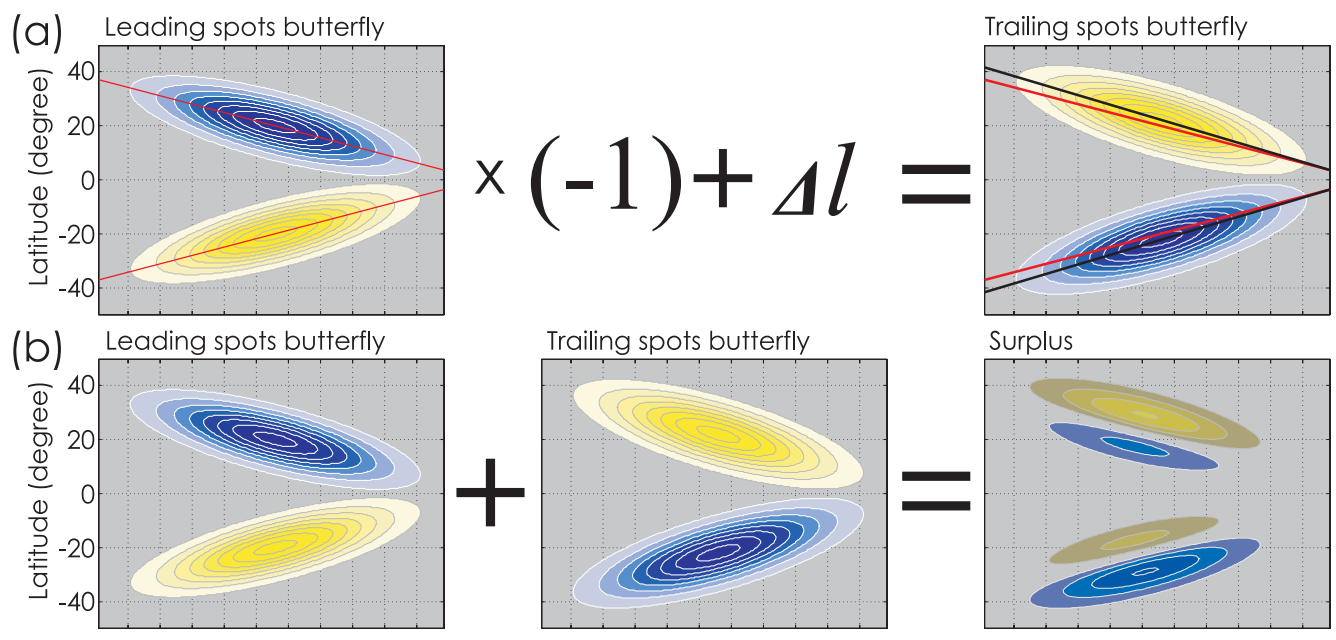

Trailing spots butterfly
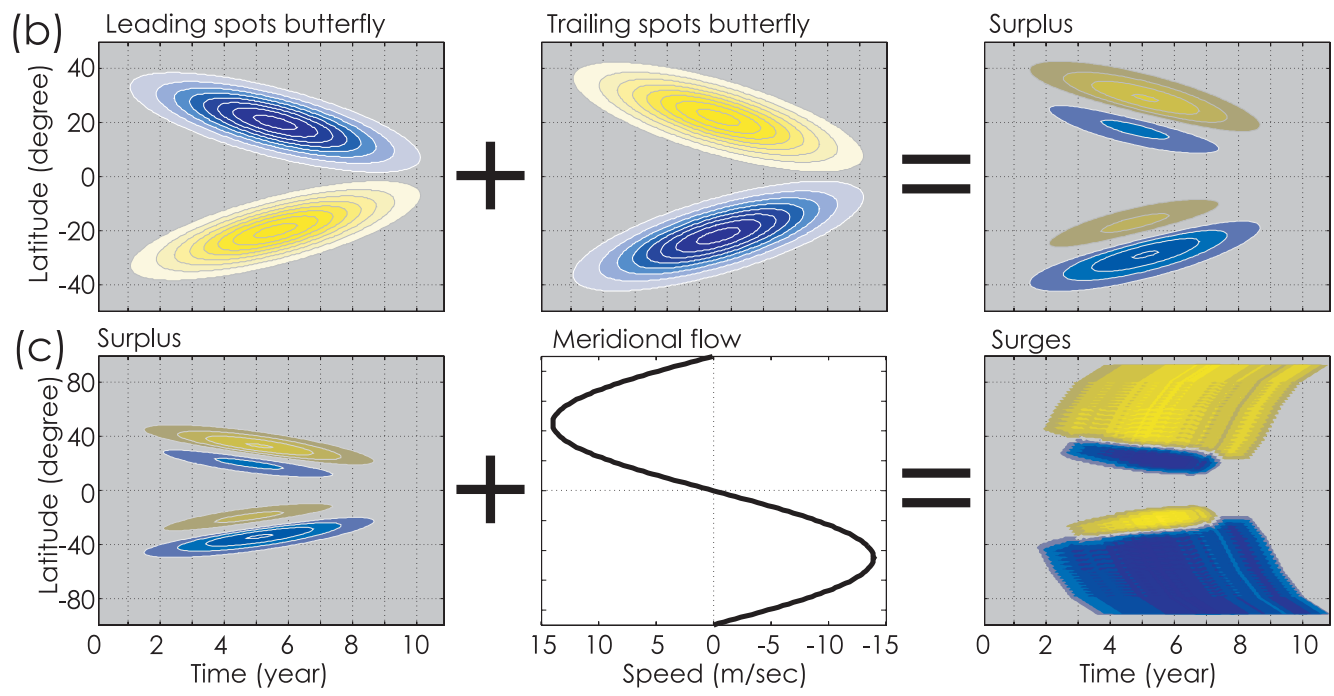

Figure 1. Simulation scheme. Yellow and blue colours define polarities. (a): Creating trailing spots butterfly from the leading one. (b): Calculating surplus. (c): Poleward streams reconstruction. 
We introduce a slight imbalance between leading and trailing PDFs (less than 10\% for benefit of total flux of trailing spots in each hemisphere, while total flux over the whole solar surface is zero). Such an imbalance can be explained by transequatorial interconnections of the rising magnetic tubes. Thus, we suggest slight predominance of new (trailing polarity) flux in each hemisphere, while total flux over the whole solar surface is zero.

Figure 1(b) shows the so-called imbalanced "flux surplus" as a result of summation of different in sign leading and trailing PDFs in hemispheres. Due to slight dominance of the trailing polarity flux over the leading one, the total net surplus has new polarity sign in each hemisphere. Figure 1(c) shows reconstruction of poleward streams by means of the meridional flow transport of imbalanced flux surplus. Imbalanced flux surplus of leading polarity is evident at low latitudes (Fig.1c, rightmost), that is also observed as photospheric magnetic field pattern. Mathematically we processed matrixes, at each step the following operation is completed:

$$
\begin{aligned}
& S_{\text {Result }}\left(\theta_{n+1}, t_{n+1}\right)=S\left(\theta_{n+1}, t_{n+1}\right)+S\left(\theta_{n}, t_{n}\right) ; \\
& v\left(\theta_{n}, t_{n}\right)=\frac{\Delta \theta}{\Delta t}=\frac{\theta_{n+1}-\theta_{n}}{\Delta t},
\end{aligned}
$$

where $S(\theta, t)$ is dimensionless value of surplus at latitude $\theta$ in point of time $t, \Delta \theta$ latitudinal drift of surplus per one Carrington rotation $\Delta t$, and $v(\theta, t)$ - the meridional flow speed.

(a)
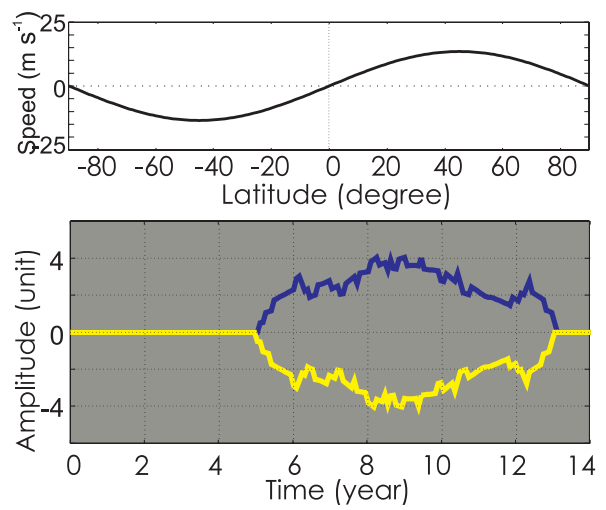

(C)
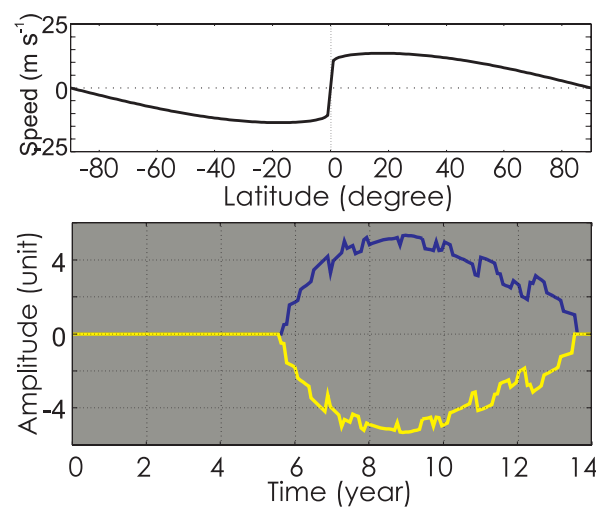

(b)
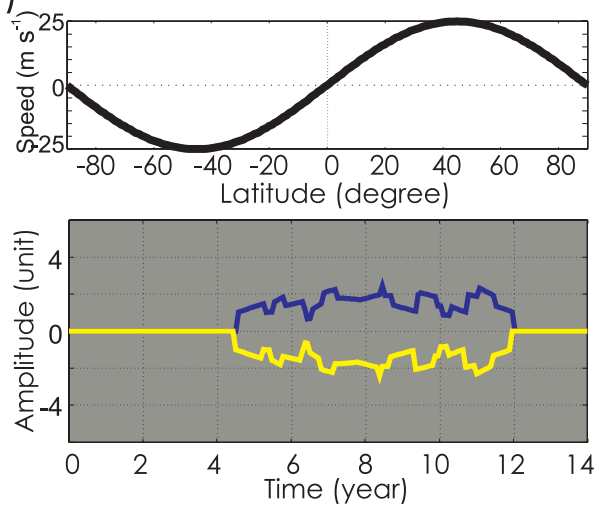

(d)
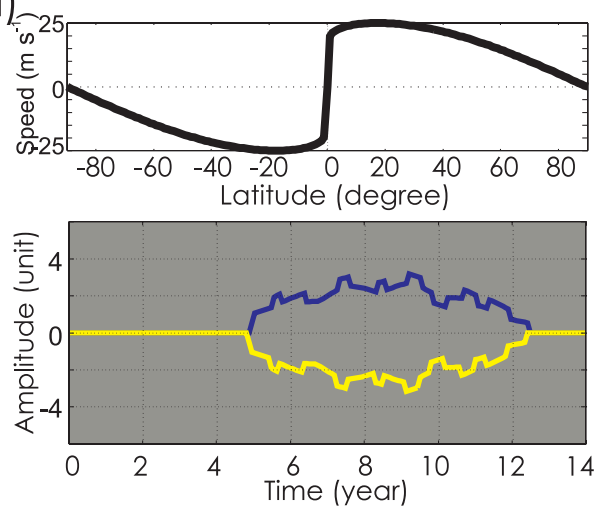

Figure 2. Various latitudinal profiles of meridional flow velocity and resulted polar magnetic field. (a) and (c): $v_{\text {max }} \simeq 13 m s^{-1}$. (b) and (d): $v_{\text {max }} \simeq 26 m s^{-1}$. 
Figure 2 demonstrates results of simulated polar magnetic field versus different meridional velocity profiles. The top picture of each of the four panels (Fig.2a-d) is the latitudinal profile of the meridional flow at the solar surface. Profiles have different maximal values $v_{\text {max }} \simeq 13 \mathrm{~ms}^{-1}$ and $v_{\max } \simeq 26 \mathrm{~ms}^{-1}$ (corresponding to $2.7 \mathrm{deg} / \mathrm{rot}$ and $5 \mathrm{deg} / \mathrm{rot}$ ), and at different latitudes 35-50deg (van Ballegooijen, Cartledge, \& Priest 1998; Schrijver \& Title 2001; Hathaway \& Rightmire 2010, Jiang et al. 2011; Hathaway \& Rightmire 2011) or 10-20deg (DeVore \& Sheeley 1987; Wang, Robbrecht, \& Sheeley 2009; Dikpati, Gilman, \& Ulrich 2010). The bottom picture of each of the four pannels (Fig.2a-d) displays the response of polar field. The faster meridional flow reduces the polar field. The same conclusion was done by Wang, Lean, \& Sheeley (2002a); Wang, Sheeley, \& Lean (2002b); Schrijver \& Liu (2008); Wang, Robbrecht, \& Sheeley (2009); Hathaway \& Rightmire (2011); Nandy, Muñoz-Jaramillo, \& Martens (2011). As well a shape of the latitudinal profile of meridional flow influences on a magnitude of simulated polar field. However results depend on the size of mesh grid. Further investigations are required.

\section{Results}

Figure 3(a) shows reconstructed magnetic field surges from RGO/USAF/NOAA daily sunspot observations (http://solarscience.msfc.nasa.gov/greenwch.shtml). Yellow and blue define polarities. In our simulations each surge reaches polar latitudes. Figure 3(b) demonstrates values of $S(\theta, t)$ at latitudes $80^{\circ}$ and $-80^{\circ}$. According to Wilcox

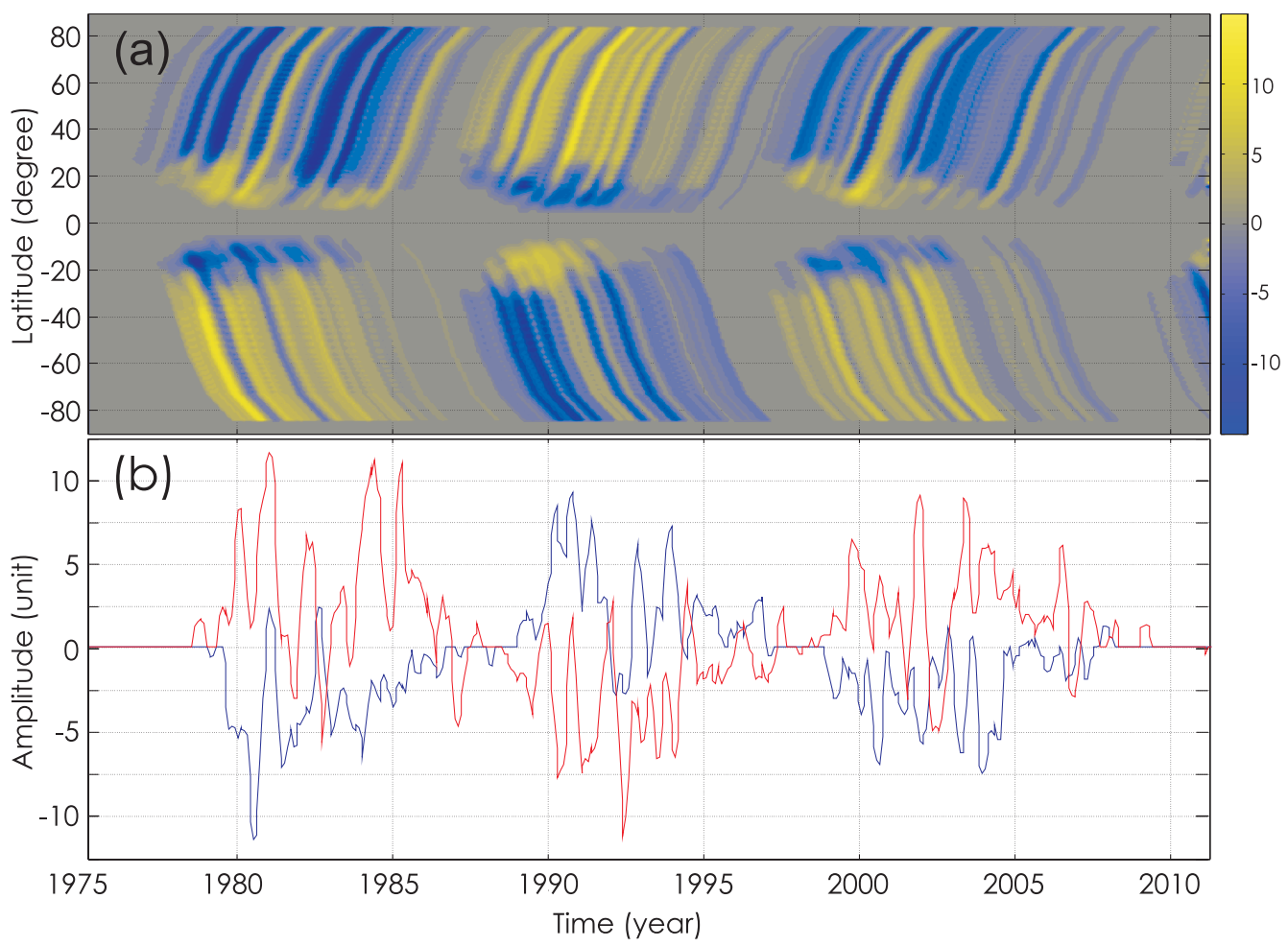

Figure 3. (a): Reconstruction of poleward magnetic field streams from real sunspot data for 1975-2011. (b): Simulated polar magnetic field in dimensionless values for cycles 21-23. 
Solar Observatory (http://wso.stanford.edu/) the reversals of polar field occurred near 1980, 1990, and 2000. According to Fig. 3(b) these years correspond to the first strong magnetic streams reaching polar latitudes. Also from WSO observations it is known that magnitude of polar field in the northern hemisphere gradually decreases from cycle 21 to cycle 23, while for the southern hemisphere the polar field in cycle 22 was slightly stronger with respect to the polar field in cycle 21 , and finally during cycle 23 the polar field was weaker than during preceding cycles. Similar regularity is seen in Fig. 3(b).

\section{Conclusion}

We have proposed a method to reconstruct magnetic field surges to the poles from sunspot impulses. We performed simulations in terms of probability density function without direct diffusion assignment. The slightly different time-latitude positions of distributions of leading and trailing activities of BMRs produces the so-called surplus of imbalanced flux. We determine a slight imbalance between leading and trailing PDFs (less than $10 \%$ for benefit of total flux of trailing spots in each hemisphere). Such an imbalance can be explained by transequatorial loops. Finally, a multiple surges to the poles can be modeled as transport of magnetic flux imbalance by meridional convection from active regions.

\section{Acknowledgments}

We thank Dr. Robert Cameron, from the Max Planck Institute for Solar System Research, for providing useful discussion and comments.

\section{References}

Antalová, A. \& Gnevyshev, M. N. 1983, Contributions of the Astronomical Observatory Skalnate Pleso 11, 63

Cowling, T. G. 1965, in: R. Lust (eds.) Stellar and Solar Magnetic Fields, Proc. IAU Symposium No. 22 (North-Holland Pub. Co., Amsterdam), p. 405

DeVore, C. R. \& Sheeley, N. R., Jr. 1987, Solar Phys. 108, 47

Dikpati, M., Gilman, P. A., \& Ulrich, R. K. 2010, ApJ 722, 774

Gnevyshev, M. N. 1938, Izvestiya Glavnoj Astronomicheskoj Observatorii v Pulkove 16, 36

Hale, G. E., Ellerman, F., Nicholson, S. B., \& Joy, A. H. 1919, ApJ 49, 153

Hathaway, D. H. 2010, Living Rev. Solar Phys. 7, 1

Hathaway, D. H. \& Rightmire, L. 2010, Science 327, 1350

Hathaway, D. H. \& Rightmire, L. 2011, ApJ 729, 80

Jiang, J., Cameron, R. H., Schmitt, D., \& Schüssler, M. 2011, Space Sci. Revs 136

Mackay, D. H., Priest, E. R., \& Lockwood, M. 2002, Solar Phys. 207, 291

Nandy, D., Muñoz-Jaramillo, A., \& Martens, P. C. H. 2011, Nature 471, 80

Schrijver, C. J. \& Liu, Y. 2008, Solar Phys. 252, 19

Schrijver, C. J. \& Title, A. M. 2001, ApJ 551, 1099

Stenflo J. O. \& Kosovichev A. G. 2012, ApJ 745, 129

van Ballegooijen, A. A., Cartledge, N. P., \& Priest, E. R. 1998, ApJ 501, 866

Wang, Y.-M., Lean, J., \& Sheeley, N. R., Jr. 2002a, Ap. Lett. 577, L53

Wang, Y.-M., Nash, A. G., \& Sheeley, N. R. Jr. 1989, ApJ 347, 529

Wang, Y.-M., Robbrecht, E., \& Sheeley, N. R., Jr. 2009, ApJ 707, 1372

Wang, Y.-M., Sheeley, N. R., \& Jr., Lean, J. 2002b, ApJ 580, 1188

Wang, Y.-M. \& Sheeley N. R., Jr. 1989, Solar Phys. 124, 81 
Wang, Y.-M. \& Sheeley, N. R., Jr. 1991, ApJ 375, 761

Zolotova, N. V. \& Ponyavin, D. I. 2012a, Astron. Rep. 56, 250

Zolotova, N. V. \& Ponyavin, D. I. 2012b, in: C.H. Mandrini \& D.F. Webb (eds.), Comparative Magnetic Minima: Characterizing quiet times in the Sun and stars, Proc. IAU Symposium No. 286 (Cambridge University Press), p. 88 